\title{
Associative detachment of rubidium hydroxide
}

\author{
Jason N. Byrd, ${ }^{1,2, *}$ H. Harvey Michels, ${ }^{1}$ John A. Montgomery, Jr., ${ }^{1}$ and Robin Côté ${ }^{1}$ \\ ${ }^{1}$ Department of Physics, University of Connecticut, Storrs, CT 06269 \\ ${ }^{2}$ Quantum Theory Project, University of Florida, Gainesville, Florida 32611
}

\begin{abstract}
We performed calculations of the optimized structure, harmonic vibrational frequencies and dissociation energies of $\mathrm{RbOH}$ and its anion, and investigate the interactions between $\mathrm{Rb}$ and $\mathrm{OH}^{-}$ leading to possible associative detachment pathways. The electron affinity of RbOH was computed to be $0.2890 \mathrm{eV}$, with a bond energy of $\mathrm{Rb}+\mathrm{OH}^{-}$half that of $\mathrm{Rb}+\mathrm{OH}$. To determine other possible charge loss pathways, the $\mathrm{Rb}+\mathrm{OH}$ and $\mathrm{Rb}+\mathrm{OH}^{-}$dissociation curves were computed using couple cluster methods along all possible collisional angles. An adiabatic curve crossing between the neutral and charged molecule was found at the inner wall of the molecular potential curve for linear geometries. Associative detachment rates were estimated using the Langevin ion capture cross-section for hydroxide. We find for $v \geq 2$ an associative detachment rate of $>2 \times 10^{-9} \mathrm{~cm}^{3} \mathrm{~s}^{-1}$, while for $v=0$ and 1 no appreciable rate exists. This strong dependence on vibrational level suggests the ability to control the associative detachment rate directly.
\end{abstract}

\section{INTRODUCTION}

Advances in the formation of cold molecules have opened up avenues into many branches of the physical sciences [1, 2]. For chemical physics, applications range from precision spectroscopy [3], to the study [4, 5] and control [6] of cold chemical reactions. Other areas of physics benefit greatly from the study of cold molecules, such as condensed matter physics 7], and the search for novel quantum gases [8] and phases [9]. Oxides such as $\mathrm{PbO}$ [10], YO [11] and TiO [12] have been of interest recently for electron dipole moment measurements, direct cooling and trapping of molecules respectively, as has the $\mathrm{OH}$ radical [13]. Molecular ions have the advantage of being easily trapped and cooled using radio frequency traps and sympathetic cooling [14] while not involving an oxide radical. Recent progress on the oxide ion front has seen the co-trapping 15] of cold rubidium and hydroxide. In this work we investigate the interactions of $\mathrm{Rb}+\mathrm{OH}$, and find that it is possible to control the associative dissociation process of forming neutral rubidium hydroxyl by accessable laser controlled transitions.

\section{ELECTRONIC STRUCTURE CALCULATIONS}

Electronic structure calculations were performed on $\mathrm{RbH}, \mathrm{RbO}, \mathrm{OH}, \mathrm{RbOH}$ and associated anions using a combination of perturbation and coupled cluster theory [16]. Second order Møller-Plesset (MP2) perturbation theory and coupled cluster theory with all singles, doubles, and perturbative triples $(\mathrm{CCSD}(\mathrm{T}))$ energy and gradient calculations in this work were carried out using the CFOUR [17] and MOLPRO 2010.1 [18] quantum chemistry packages. Higher order calculations involving CCSDT and CCSDT(Q) (all triples and perturba-

\footnotetext{
*byrdja@chem.ufl.edu
}

tive quadruples respectively) were done using the MRCC program of M. Kállay [19]. For open shell systems, the spin restricted variants of these theories were used. Due to the size of rubidium, there are a number of correlation space choices available for consideration. We have adopted the same notation as Sullivan et al. [20] where valence only calculations (H:1s; O:2s2p; Rb:5s) are referred to as relaxed valence (rv). Increasing the correlation space size to involve the first set of sub-valence orbitals (H:1s; O:1s2s2p; Rb:4s4p5s) results in the relaxed innervalence (riv), while spaces including yet deeper orbitals (H:1s; O:1s2s2p; Rb:3s3p3d4s4p5s) are called riiv and so forth. Valence (rv) only calculations involving rubidium and oxygen require extra care, as the usual method of energy sorting orbitals in selecting the frozen core will fail since the energy of $2 s$ orbital of oxygen is below the $4 p$ orbital of rubidium. Failing to properly choose the core orbitals for valence calculations will lead to significant errors.

\begin{tabular}{llllll}
\hline \hline & $\mathrm{r}(\mathrm{M}-\mathrm{H})$ & $\mathrm{r}(\mathrm{M}-\mathrm{O})$ & $\mathrm{ZPE}^{\mathrm{a}}[21]$ & $\mathrm{EA}$ & $\mathrm{AE}$ \\
\hline $\mathrm{OH}$ & 0.9698 & & 8.53 & & 162.91 \\
Exp. & $0.9696[22]$ & & $8.51[21]$ & & $161.53[23]$ \\
$\mathrm{OH}^{-}$ & 0.9643 & & 8.55 & 1.8405 & 176.51 \\
Exp. & $0.9643[24]$ & & $8.51[24]$ & $1.8277[25]$ & $175.65[26]$ \\
$\mathrm{RbO}$ & & 2.3548 & 0.79 & & 102.21 \\
Exp. & & $2.2541[27]$ & $0.84[27]$ & & \\
$\mathrm{RbO}^{-}$ & & 2.2564 & 0.65 & 0.1002 & 58.90 \\
$\mathrm{RbH}^{2}$ & 2.3919 & & 1.12 & & 61.39 \\
Exp. & $2.37[28]$ & & $1.34[28]$ & & \\
RbH & & & & \\
$\mathrm{RbOH}^{-}$ & 2.5415 & & 1.66 & 0.3604 & 47.11 \\
Theory & 0.9551 & 2.3408 & 11.72 & & 291.26 \\
$\mathrm{RbOH}^{-}$ & 0.9567 & $2.472[29]$ & $11.36[29]$ & & \\
\hline \hline
\end{tabular}

a $\mathrm{ZPE} \simeq \omega_{e} / 2$.

TABLE I. Computed bond lengths, harmonic zero point energies, electron affinities (EA) and atomization energies (AE) for $\mathrm{RbOH}$, its constitutes and their anions (Units are in angstroms, electron volts and $10^{-3}$ a.u. as appropriate). 


\begin{tabular}{lrrr}
\hline \hline method & space & $\mathrm{Rb}+\mathrm{OH}^{-}$ & $\mathrm{Rb}+\mathrm{OH}$ \\
\hline $\mathrm{CCSD}(\mathrm{T}) / \mathrm{TZ}$ & riv & 75.40 & 129.25 \\
$\mathrm{CCSD}(\mathrm{T}) / \mathrm{QZ}$ & riv & 74.63 & 130.47 \\
\hline $\mathrm{CCSD}(\mathrm{T}) /$ Extrap. & riv & 74.07 & 131.36 \\
$\Delta \mathrm{CCSD}(\mathrm{T}) / \mathrm{ANO}$ & riiv & 0.25 & 0.21 \\
Total Energy & - & 74.20 & 131.54 \\
\hline \hline
\end{tabular}

TABLE II. Breakdown of the contributions of various levels of theory to the Rb-O bond energy (in $10^{-3}$ a.u.) for both neutral and charged $\mathrm{RbOH}$.

While there are many basis sets available for the first row elements, the basis set selection for rubidium is sparse. This is further complicated by the need for diffuse functions to accurately describe electron affinities [30]. Previous calculations [31, 32] involving rubidium using the Karlshruhe def2-nZVPP basis sets [33, 34] ( $\mathrm{n}=\mathrm{T}, \mathrm{Q}$ zeta quality basis sets with two extra spdf correlation polarization functions) have shown good experimental agreement for both dissociation energies and bond lengths of the $\mathrm{Rb}_{2}$ diatom. For rubidium these basis sets use the small-core ECP28MWB [35] Stuttgart pseudopotential, which removes the argon core electrons from the calculation while leaving the $4 \mathrm{~s} 4 \mathrm{p} 5 \mathrm{~s}$ electrons free for use in further correlation calculations. The addition of even tempered spdf diffuse functions to these basis sets was done to better describe the anion, while the addition of these diffuse functions has also shown to improve molecular properties [36, 37] as well. This aug-Def2-nZVPP basis set was used for rubidium in all riv electronic structure calculations in this work. To best describe the $\mathrm{OH}$ bond, the optimized aug-cc-pVnZ valence [30] and augcc-wCVnZ weighted core-valence [38] correlation basis sets were used for hydrogen and oxygen respectively.

Molecular structures were optimized using the $\operatorname{CCSD}(\mathrm{T}) /$ riv level of theory using the quadruple zeta (QZ) quality basis sets 39]. Frequency calculations at the riv $\operatorname{CCSD}(\mathrm{T})$ level of theory using the QZ basis sets were performed for each optimized structure to identify whether the structure was a transition state or a local minimum of the potential energy surface. The final ground state structure of the $\mathrm{RbOH}^{-}$ion is found to be linear, consistent with the ground state structure of the neutral molecule [29, 40]. Additionally the conformers $\mathrm{OHRb}^{-}$and $\mathrm{ORbH}^{-}$were also investigated and found to be transition states. Vibrational harmonic zeropoint energy (ZPE) corrections were computed for the final structures at the $\operatorname{CCSD}(\mathrm{T}) /$ riv level of theory using the QZ basis set. Computed bond lengths and ZPE corrections are listed in Table [.

Correlation calculations involving the riiv electrons of rubidium involve electrons dropped by the MWB pseudopotential. Because of this we perform the riiv correlation calculations all electron using the Roos atomic natural orbital (ANO) basis set [1], which was chosen for its availability for all atoms present and its noted consistency [42]. Prior to use, the basis set was completely

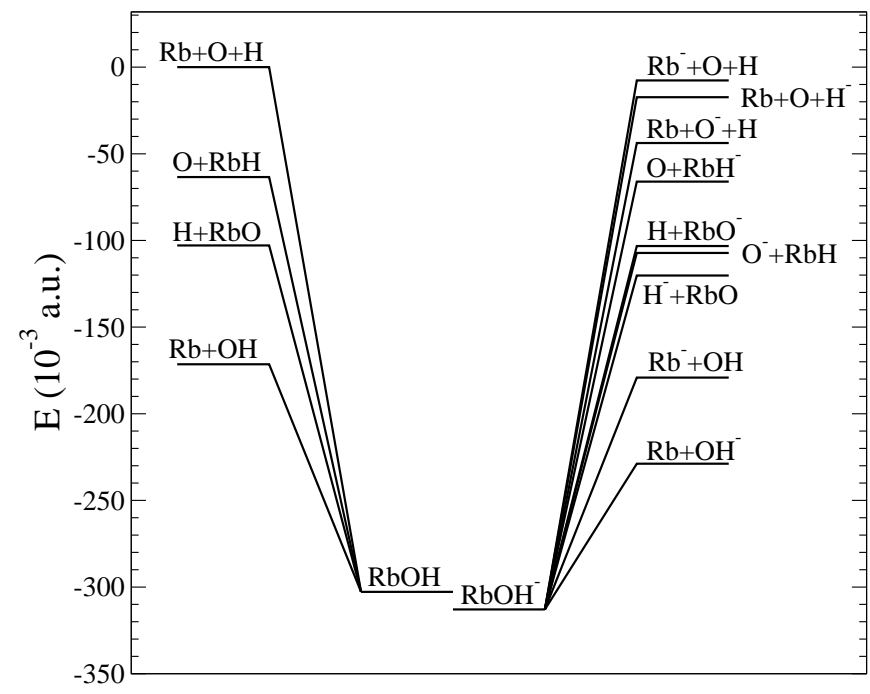

FIG. 1. Dissociation limits of rubidium hydroxyl (left) and its anion (right) up to the atomization limit. Energies are computed at the $\operatorname{CCSD}(\mathrm{T}) / \mathrm{CBS}$ level of theory.

uncontracted so as to be as flexible as possible in subsequent correlation calculations. Scalar relativistic effects were accounted for by adding in the one-electron secondorder Douglas-Kroll-Hess [43, 44] contribution. For rv and riv calculations, which use the Def2 basis sets for rubidium, the small core MWB family of pseudopotentials have been shown 35] to accurately account for the relativistic contributions to the bond length and dissociation energy.

The complete basis set limit (CBS) of the various contributions to the total energy was estimated using the two point linear extrapolation formula of Helgaker et al. [45],

$$
E_{\mathrm{CBS}}(\text { method })=\frac{n^{3} E_{n}-(n-1)^{3} E_{n-1}}{n^{3}-(n-1)^{3}}
$$

This extrapolation scheme was chosen over other more optimized schemes due to the spread of basis sets and correlation spaces used here. The final interaction energy is computed from the various contributions by the following formula

$$
E_{\text {int }}=E_{\mathrm{CBS}}(\operatorname{CCSD}(\mathrm{T}) / \text { riv })+E(\Delta \operatorname{CCSD}(\mathrm{T}) / \text { riiv })
$$

where $E(\operatorname{CCSD}(\mathrm{T}) /$ riv $)$ is the total $\operatorname{CCSD}(\mathrm{T}) /$ riv energy and

$$
\begin{aligned}
E(\Delta \operatorname{CCSD}(\mathrm{T}) / \text { riiv })= & E(\operatorname{CCSD}(\mathrm{T}) / \mathrm{ANO} / \text { riiv }) \\
& -E(\operatorname{CCSD}(\mathrm{T}) / \mathrm{ANO} / \text { riv })
\end{aligned}
$$

is the riiv contribution. Higher order triples contributions beyond the $\operatorname{CCSD}(\mathrm{T})$ level of theory were estimated by performing CCSDT/rv calculations using QZ quality basis sets. Effects of connected quadruple excitations, known [26] to be important for $\mathrm{OH}^{-}$, were estimated using $\operatorname{CCSDT}(\mathrm{Q}) / \mathrm{rb}$ with the triple zeta (TZ) quality 


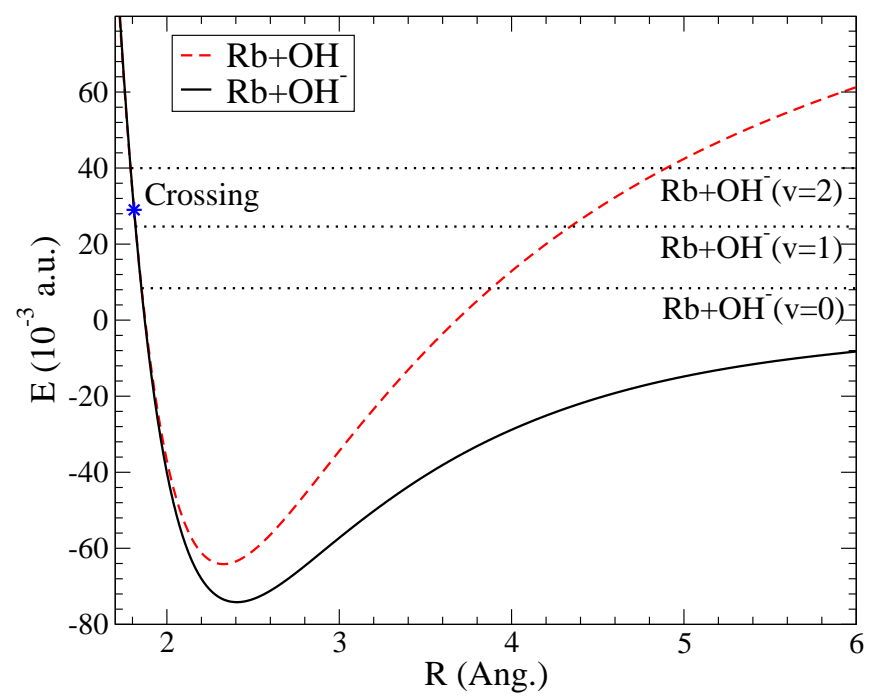

FIG. 2. Potential energy curve of $\mathrm{Rb}+\mathrm{OH}$ and its anion computed along the $\mathrm{C}_{\infty v}$ axis at the $\operatorname{CCSD}(\mathrm{T}) / \mathrm{CBS}$ level of theory. The $\mathrm{OH}$ bond length is held fixed over the entire curve for simplicity. Also shown are the energy asymptotes for various $\mathrm{OH}^{-}$vibrational levels.

basis sets. It was found that the contribution of these higher order terms to the final electron affinity (EA) are small $\left(<5 \times 10^{-5}\right.$ a.u. $)$ due to cancellation. While the riiv correlation contribution is similarly small for the EA of $\mathrm{RbOH}$ at the equilibrium geometry, it becomes more significant for much shorter Rb-O bond lengths (further discussed below). In Table II] the contributions of each of these corrections to the Rb-O bond energy are listed. Molecular bond lengths, electron affinities (EA) and atomization energies (AE) (including the harmonic ZPE correction) are reported in Table \. The excellent agreement with available experimental bond lengths, harmonic frequencies and electron affinities leads us to expect comparable accuracy for the $\mathrm{RbOH}$ complex.

\section{COMPUTATIONAL RESULTS AND DISCUSSION}

The EA and similar geometric structure of rubidium hydroxyl and its anion, along with the large difference in neutral and anion dissociation limits illustrated in Fig. 1. does not suggest immediately a charge loss pathway. In fact, at 300 Kelvin no other dissociation channels are energetically accessible. This is clearly illustrated in Fig. 2 where the minimum energy dissociation path of $\mathrm{Rb}$ for both $\mathrm{RbOH}$ and its anion are computed at the fixed $\mathrm{OH}$ bond $\operatorname{CCSD}(\mathrm{T}) / \mathrm{riv} / \mathrm{CBS}$ level of theory. It can be seen that the neutral and anion curves do not cross at any point along the incoming path. However it should be noticed that the inner wall of these curves become nearly degenerate at this level of theory. We examine the inner wall more closely, by relaxing the $\mathrm{OH}$ bond at each $\mathrm{Rb}-\mathrm{O}$ distance using MP2 gradients (as noted pre-

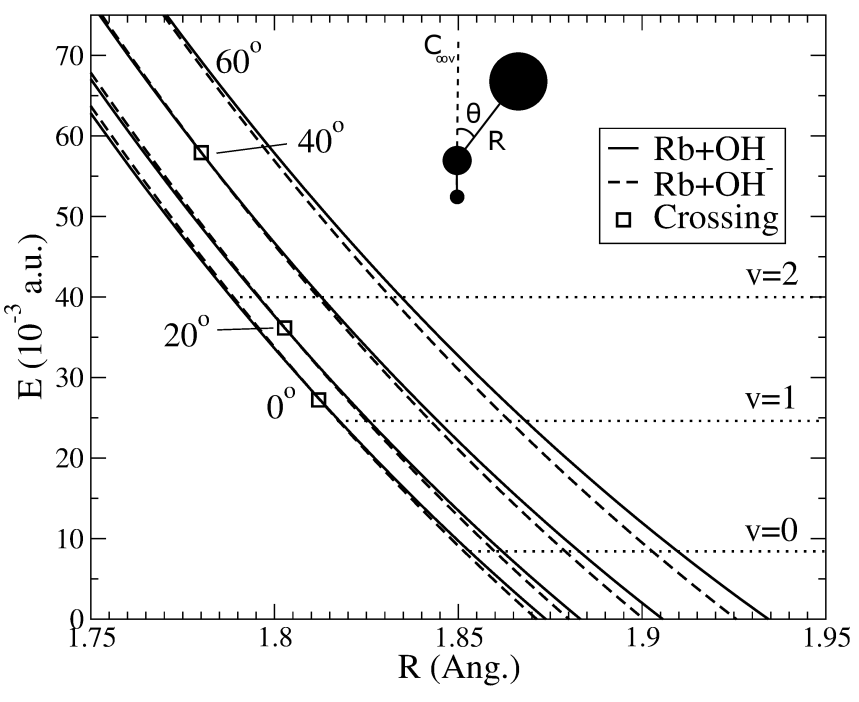

FIG. 3. Inner wall potential energy curve of $\mathrm{Rb}+\mathrm{OH}$ and its anion computed for various collisional angles at the $\operatorname{CCSD}(\mathrm{T}) / \mathrm{CBS}$ level of theory with the $\mathrm{OH}$ bond length relaxed at each point of the curve. The sketch defines the angle $\theta$ of the $\mathrm{Rb}$ (largest circle) approach to $\mathrm{OH}^{-}$centered on the oxygen (with $\mathrm{O}$ larger than $\mathrm{H}$ ) from the $\mathrm{C}_{\infty v}$ axis.

viously, MP2 bond lengths are very close to $\mathrm{CCSD}(\mathrm{T})$ bond lengths), and find that indeed the neutral and anion curves cross at $\mathrm{r}(\mathrm{Rb}-\mathrm{O}) \sim 1.81 \AA$ with a barrier height of $V_{c}(0) \sim 3.0 \times 10^{-2}$ a.u. above the $\mathrm{Rb}+\mathrm{OH}^{-}$dissociation limit, as illustrated in Fig. 3. This crossing energy $V_{c}(\theta)$ also includes the $\operatorname{CCSD}(\mathrm{T}) /$ riiv correction which provides $\sim 4 \times 10^{-4}$ a.u. to the final barrier height. This crossing is energetically accessible if the internal rotational and vibrational energy of $\mathrm{OH}^{-}$is taken into account. In fact, it is well known that producing rotationally and vibrationally cool $\mathrm{OH}^{-}$is difficult experimentally 24].

The height of $V_{c}(\theta)$ for geometries other than the linearly minimum energy approach was also investigated. It was found that for small angle approaches, relative to the equilibrium geometry, the crossing remains relatively flat, while for angles greater than 40 degrees the barrier rapidly increases in height until it is completely energetically inaccessible (see Fig. 33).

To evaluate the associative detachment rate coefficient, while accounting for the dependence with the angle of approach, we assume that $\mathrm{OH}^{-}$, in its internal rovibrational state $(v, J)$, is rotating sufficiently fast during its encounter with $\mathrm{Rb}$. We then average the angular dependence of $V_{c}(\theta)$ to obtain an effective angular phase space $\rho_{c}(v, J, \varepsilon)$ where the curve crossing is accessible for a given collision energy $\varepsilon$,

$$
\rho_{c}(v, J, \varepsilon)=\frac{1}{2} \int_{0}^{\pi} d(\cos \theta) \Theta\left[\left(\varepsilon-V_{c}(\theta)+T(v, J)\right] .\right.
$$

Here, the prefactor $\frac{1}{2}$ arises from the azimuthal angle integration, $\Theta(\cdots)$ is a Heaviside step function representing the height of the crossing as a function of the 
collision angle and for a given collision energy $\varepsilon$, and $T(v, J)=G(v)+F_{v}(J)$ is the internal rotation-vibration energy of the $\mathrm{OH}^{-}$fragment in its $v$ 'th vibrational and $J$ 'th rotational state, which we take to follow a Dunham series [46]. The integral over $\theta$ involving $V_{c}(\theta)$ is performed numerically, with representative values presented in Table III for a few $J$ 's of $v=0, \ldots, 3$ at $\varepsilon / k_{B}=300 \mathrm{~K}$ ( $k_{B}$ is the Boltzmann constant). We find that $\rho_{c}(v, J, \varepsilon)$ is negligible for $v=0$ and $v=1$ for low $J$ 's, and becomes more significant, reaching the range of $10-20 \%$ for $v \geq 2$ in this table.

Using this approximation, we estimate the total cross section for associative detachment $\sigma_{\text {tot }}(v, J, \varepsilon)$ by multiplying the Langevin cross section $\sigma_{L}(\varepsilon)$ for entering the inner region of the $\mathrm{Rb}+\mathrm{OH}^{-}$curve (where the process can take place with assumed unit probability) by the fraction of angular phase space $\rho_{c}(v, J, \varepsilon)$ allowing the process (i.e. when the curve crossing is accessible)

$$
\sigma_{\text {tot }}(v, J, \varepsilon)=\sigma_{L}(\varepsilon) \rho_{c}(v, J, \varepsilon)
$$

Here, $\sigma_{L}(\varepsilon)$ depends on the static dipole polarizability $\alpha_{d}$ of the neutral monomer (with $\alpha_{d}=318.6$ for $\mathrm{Rb}$ 47\|), but not on the inner part of the potential curve [48, 49]

$$
\sigma_{L}(\varepsilon)=\pi \sqrt{\frac{2 \alpha_{d}}{\varepsilon}}
$$

A thermal rate constant $k_{\text {ad }}(v, J)$ for the associative detachment for $\mathrm{OH}^{-}$initially in a specific $(v, J)$ state is obtained by averaging $v \sigma_{\text {tot }}$ over a Maxwell distribution of velocity $v$ characterized by a (translational) temperature $T$, namely

$$
k_{\mathrm{ad}}(v, J)=\sqrt{\frac{2 k_{B} T}{\pi \mu}} \int_{0}^{\infty} d x x e^{-x} \sigma_{\mathrm{tot}}\left(v, J, x k_{B} T\right)
$$

where $x=\varepsilon / k_{B} T$, and $\mu$ is the reduced mass of the colliding partners (here $\mathrm{Rb}$ and $\mathrm{OH}^{-}$). The distribution of rotational states $J$ is assumed to be thermalized against the collision energy, which gives the vibrational state rate constant

$$
k_{\mathrm{ad}}(v)=\frac{1}{Q_{\mathrm{rot}}} \sum_{J}^{J_{\mathrm{max}}} k_{\mathrm{ad}}(v, J) \exp \left(-\frac{F_{v}(J)}{k_{B} T}\right),
$$

TABLE III. Representative values of the accessible angular space, Eq. (44), for various vibrational and rotational states for a collisional energy of 300 Kelvin.

\begin{tabular}{rrrrr}
\hline \hline & \multicolumn{4}{c}{$\rho_{c}(v, J, 300 K) \times 100$} \\
$J$ & $v=0$ & $v=1$ & $v=2$ & $v=3$ \\
\hline 0 & 0.00 & 0.00 & 5.38 & 11.00 \\
5 & 0.00 & 0.00 & 6.56 & 12.07 \\
10 & 0.00 & 3.16 & 9.53 & 14.80 \\
15 & 0.89 & 8.11 & 14.00 & 18.92 \\
\hline \hline
\end{tabular}

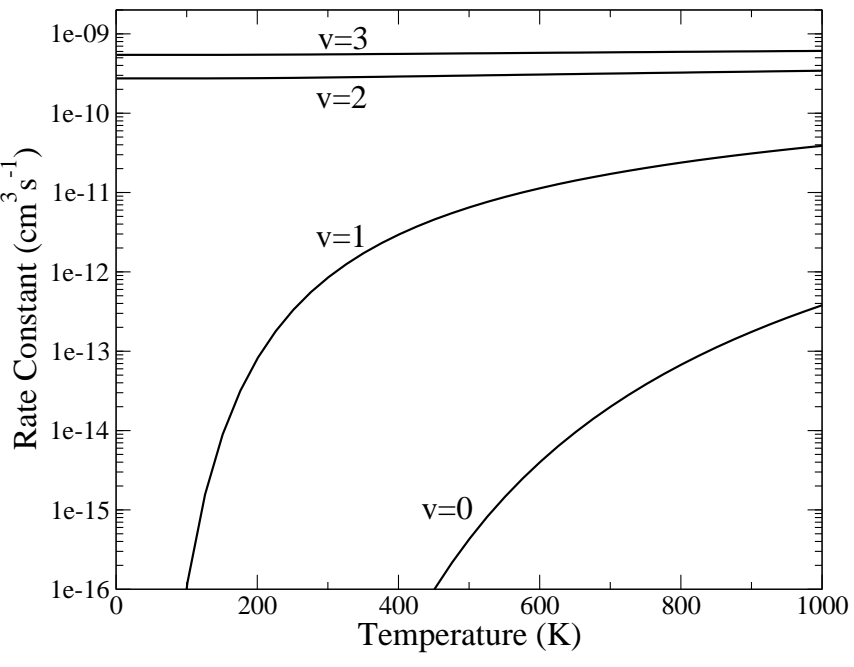

FIG. 4. Ion capture rate coefficient as a function of the collisional temperature of the $\mathrm{OH}^{-}$complex for the first four vibrational levels.

with the rotational partition function given by

$$
Q_{\mathrm{rot}}=\sum_{J}^{J_{\max }} \exp \left(-\frac{F_{v}(J)}{k_{B} T}\right)
$$

Here $J_{\max }$ is the maximum rotational state taken in the series. To evaluate Eq. (8) we use the spectroscopic constants of Rosenbaum et al. [24] in $T(v, J)$ [50] and choose $J_{\max }$ such that the thermodynamic contribution of that rotational state is negligible (see Fig. 5] a)). A value of $J_{\max }=15$ was found to be more than adequate to converge the sums in Eqs. (8)-(9) even for very high temperatures. The rate constant (8) was evaluated numerically for the first three vibrational levels of $\mathrm{OH}^{-}$as a function of the collisional temperature, the results of which are plotted in Fig. 4. The rate constant for $v=0$ and $v=1$ is found to be much lower than a recent experimental value [15], but becomes comparable for $v=2$, as expected considering the energetics of the collision. It should be noted that for $v=2$ and higher, the incoming collisional energy is above the curve crossing threshold, and so the rate coefficient is a nearly constant as expected.

As these collisions involve rotationally and vibrationally excited states of a polar molecule, it is important to characterize the lifetime of these states in the absence of collisions. To do this we have computed the $\mathrm{OH}^{-}$potential energy curve near the equilibrium geometry at the $E_{\mathrm{CBS}}(\mathrm{CCSD}(\mathrm{T}) /$ riv $)$ level of theory as well as the static dipole moment $\mathcal{D}(R)$ along this curve at the $\operatorname{CCSD}(\mathrm{T}) / \mathrm{rv}$ level of theory using the aug-cc-pVQZ basis set. We find that the resulting dipole moment function is in good agreement [51] with the original work of Werner et al. [52]. The spontaneous emission rate for a given initial rotational and vibrational state $J^{\prime}, v^{\prime}$ radiating to all possible lower energy rotational states is (in atomic 


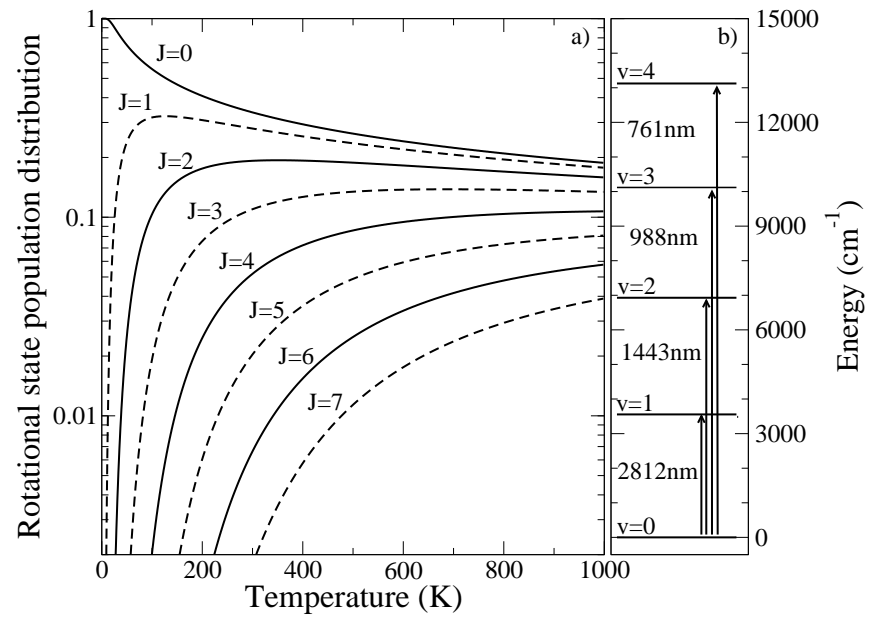

FIG. 5. a) Rotational state population distribution as a function of temperature for the $v=0$ state of $\mathrm{OH}^{-}$. Effects on the rotational distribution as a function of vibrational level are quantitative only and vary on the order of $3 \%$. b) Rotationless vibrational level energies, $T(v, 0)$, for $\mathrm{OH}^{-}$and transition wavelengths from $v=0$ to various excited vibrational levels.

units [53]) given by

$$
\begin{array}{r}
A_{v^{\prime} \rightarrow v}\left(J^{\prime}\right)=\sum_{J} \frac{4}{3} \alpha^{3}\left(T\left(v^{\prime}, J^{\prime}\right)-T(v, J)\right)^{3} \times \\
\left\langle v^{\prime} ; J^{\prime} M^{\prime} \Omega^{\prime}|\mathcal{D}(R)| J M \Omega ; v\right\rangle,
\end{array}
$$

where $|v\rangle$ is the vibrational wave function for the $v$ th level and $|J M \Omega\rangle$ is the rigid-rotor wavefunction [54]. We have tabulated our results for $A_{J^{\prime}, v^{\prime} \rightarrow v}$ in Table IV for all $J^{\prime}$ up to $J_{\max }$, for the various vibrational transitions for $v=0$

TABLE IV. Spontanious emission rotational rate coefficients, Eq. (10), for $\mathrm{OH}^{-}$in various starting rotational and vibrational levels. Units are in inverse seconds and $[n]$ represents

\begin{tabular}{|c|c|c|c|c|c|c|}
\hline \multirow[b]{2}{*}{$J^{\prime}$} & \multicolumn{6}{|c|}{$A_{v^{\prime} \rightarrow v}\left(J^{\prime}\right)\left(\mathrm{s}^{-1}\right)$} \\
\hline & $1 \rightarrow 0$ & $2 \rightarrow 0$ & $2 \rightarrow 1$ & $3 \rightarrow 0$ & $3 \rightarrow 1$ & $3 \rightarrow 2$ \\
\hline$\overline{0}$ & $6.87[1]$ & $5.16[1]$ & $1.22[2]$ & $2.19[1]$ & $1.36[0$ & $1.75[2$ \\
\hline 1 & $2.36[2]$ & $1.78[0]$ & $4.18[2]$ & $7.54[1]$ & $4.68[0$ & $6.00[2$ \\
\hline 2 & $4.34[2]$ & $3.26[0]$ & $7.68[2]$ & $1.38[0]$ & $8.58[0$ & $1.10[3$ \\
\hline 3 & $6.31[2]$ & $4.72[0]$ & $1.12[3]$ & $2.00[0]$ & $1.24[1$ & $1.61[3$ \\
\hline 4 & $8.30[2]$ & $6.20[0]$ & $1.47[3]$ & $2.63[0]$ & $1.64[1$ & $2.12[3$ \\
\hline 5 & $1.03[3]$ & $7.70[0]$ & $1.83[3]$ & $3.27[0]$ & $2.03[1$ & $2.64[3$ \\
\hline 6 & $1.24[3]$ & $9.23[0]$ & $2.21[3]$ & $3.91[0]$ & $2.44[1$ & $3.19[3$ \\
\hline 7 & $1.46[3]$ & $1.08[1]$ & $2.60[3]$ & $4.58[0]$ & $2.86[1$ & $3.76[3$ \\
\hline 8 & $1.69[3]$ & $1.24[1]$ & $3.01[3]$ & $5.26[0]$ & $3.29[1$ & $4.36[3$ \\
\hline 9 & $1.92[3]$ & $1.41[1]$ & $3.43[3]$ & $5.97[0]$ & $3.74[1$ & $4.98[3$ \\
\hline 10 & $2.17[3]$ & $1.59[1]$ & $3.88[3]$ & $6.71[0]$ & $4.20[1$ & $5.65[3$ \\
\hline 11 & $2.43[3]$ & $1.77[1]$ & $4.36[3]$ & $7.47[0]$ & $4.69[1$ & $6.36[3$ \\
\hline 12 & $2.70[3]$ & $1.96[1]$ & $4.86[3]$ & $8.27[0]$ & $5.20[1$ & $7.11[3$ \\
\hline 13 & $3.00[3]$ & $2.16[1]$ & $5.40[3]$ & $9.10[0]$ & $5.74[1$ & $7.91[3$ \\
\hline 14 & $3.30[3]$ & $2.36[1]$ & $5.97[3]$ & $9.97[0]$ & $6.30[1$ & $8.77[3$ \\
\hline 15 & $3.63[3]$ & $2.58[1]$ & $6.57[3]$ & $1.09[1]$ & $6.89[1$ & $9.69[3$ \\
\hline
\end{tabular}
$\times 10^{n}$.
TABLE V. Temperature dependent vibrational state lifetimes averaged over inital rotational states. Units are in kelvin and miliseconds.

\begin{tabular}{ccrr}
\hline \hline temperature & $v^{\prime}=1$ & $v^{\prime}=2$ & $v^{\prime}=3$ \\
\hline 10 & 14.5 & 8.17 & 5.67 \\
100 & 9.94 & 5.60 & 3.88 \\
300 & 6.98 & 3.93 & 2.72 \\
600 & 5.44 & 3.06 & 2.12 \\
\hline \hline
\end{tabular}

through $v=3$ (see Fig. 5 b)). Given an inital vibrational state, the temperature dependent lifetime averaged over rotational level is

$$
\tau_{v^{\prime}}=\frac{1}{Q_{\text {rot }}} \sum_{v} \sum_{J}^{J_{\max }} \frac{\exp \left(-F_{v}(J) / k_{B} T\right)}{A_{v^{\prime} \rightarrow v}\left(J^{\prime}\right)} .
$$

In Table $\nabla$ the lifetimes for the first 3 excited vibrational levels are listed at several representative temperatures.

\section{CONCLUSIONS}

We have computed $\operatorname{CCSD}(\mathrm{T})$ ab initio potential energy curves for the $\mathrm{Rb}+\mathrm{OH}$ and $\mathrm{Rb}+\mathrm{OH}^{-}$systems, and found a neutral-ion curve crossing along the inner wall of the potential energy curve for collinear geometries of $\mathrm{RbOH}$. Further investigation of the potential energy surface shows that this crossing is highly dependent on collisional angle and is accessible to reasonable scattering energies only for angles near the collinear geometry. Furthermore, this crossing lies above the $\mathrm{OH}^{-}(v=0)$ collisional threshold, and so is expected to have a negligible contribution to the long-term co-trapping of rubidium and hydroxide. Using the Langevin capture crosssection, we evaluate the associative detachment rate for the first few vibrationally excited states of hydroxide colliding with rubidium and find an appreciable rate coefficient $>2 \times 10^{-9} \mathrm{~cm}^{3} \mathrm{~s}^{-1}$ for hydroxide vibrational levels $v \geq 2$. Lifetimes for these vibrationally excited hydroxide molecules are computed, and found to be on the order of $5 \mathrm{~ms}$. With transitions between $v=0$ and $v \geq 2$ (see Fig. (5 b)) in the near infrared, it is possible to directly control access to the associative recombination pathway that forms neutral $\mathrm{RbOH}$.

\section{ACKNOWLEDGMENTS}

We would like to thank M. Weidemüller for many helpful conversations during the course of this work. The work of J.N.B. was partially supported by the US Department of Energy, Office of Basic Energy Sciences, and the work of R.C. by the National Science Foundation Grant No. PHY 1101254. 
[1] L. Carr, D. DeMille, R. Krems, and J. Ye, New J. Phys. 11, 055049 (2009).

[2] O. Dulieu, R. Krems, M. Weidemüller, and S. Willitsch, Phys. Chem. Chem. Phys. 13, 18703 (2011).

[3] D. DeMille, S. Sainis, J. Sage, T. Bergeman, S. Kotochigova, and E. Tiesinga, Phys. Rev. Lett. 100, 043202 (2008).

[4] M. de Miranda, A. Chotia, B. Neyenhuis, D. Wang, G. Quéméner, S. Ospelkaus, J. Bohn, J. Ye, and D. Jin, Nature Physics 7, 502 (2011).

[5] B. Sawyer, B. Stuhl, M. Yeo, T. Tscherbul, M. Hummon, Y. Xia, D. P. J. Klos, J. Doyle, and J. Ye, Phys. Chem. Chem. Phys. 13, 19059 (2011).

[6] G. Quéméner and P. Julienne, Chem. Rev. 112, 4949 (2012).

[7] A. Micheli, G. K. Brennen, and P. Zoller, Nat. Phys. 2, 341 (2006).

[8] L. Santos, G. Shlyapnikov, P. Zoller, and M. Lewenstein, Phys. Rev. Lett. 85, 1791 (2000).

[9] A. Recati, P. Fedichev, W. Zwerger, and P. Zoller, Phys. Rev. Lett. 90, 020401 (2003).

[10] D. DeMille, F. Bay, S. Bickman, D. Kawall, D. Krause, S. Maxwell, and L. Hunter, Phys. Rev. A 61, 052507 (2000).

[11] M. Hummon, M. Yeo, B. Stuhl, A. Collopy, Y. Xia, and J. Ye, Phys. Rev. Lett. 110, 143001 (2013).

[12] B. Stuhl, B. Sawyer, D. Wang, , and J. Ye, Phys. Rev. Lett. 101, 243002 (2008).

[13] B. Stuhl, M. Hummon, M. Yeo, G. Quéméner, J. Bohn, and J. Ye, Nature 492, 396 (2013).

[14] S. Schiller and B. Roth, in Cold Molecules, Theory, Experiment and Applications, edited by R. V. Krems, W. C. Stwalley, and B. Friedrich (CRC Press, Boca Raton, 2009) Chap. 18.

[15] J. Deiglmayr, A. Göritz, T. Best, M. Weidemüller, and R. Wester, Phys. Rev. A 86, 043438 (2012).

[16] K. Raghavachari, G. W. Trucks, J. A. Pople, and M. Head-Gordon, Chem. Phys. Lett. 157, 479 (1989).

[17] J. F. Stanton, J. Gauss, M. E. Harding, P. G. Szalay, A. A. Auer, R. J. Bartlett, U. Benedikt, C. Berger, D. E. Bernholdt, Y. J. Bomble, O. Christiansen, M. Heckert, O. Heun, C. Huber, T.-C. Jagau, D. Jonsson, J. Juslius, K. Klein, W. J. Lauderdale, D. A. Matthews, T. Metzroth, D. P. O'Neill, D. R. Price, E. Prochnow, K. Ruud, F. Schiffmann, S. Stopkowicz, J. Vzquez, F. Wang, and J. D. Watts, "CFOUR, coupled-cluster techniques for computational chemistry, a quantum-chemical program package," Http://www.cfour.de.

[18] H.-J. Werner, P. J. Knowles, F. R. Manby, M. Schütz, et al., "Molpro, version 2010.1, a package of ab initio programs," (2010), see http://www.molpro.net

[19] M. Kállay and P. Surján, J. Chem. Phys. 115, 2945 (2001).

[20] M. Sullivan, M. Iron, P. Redfern, J. Martin, L. Curtiss, and L. Radom, J. Phys. Chem. A 107, 5617 (2003).

[21] M. Abrams, S. Davis, M. Rao, and R. E. Jr., Astrophys. J. Suppl. Ser. 93, 351 (1994).

[22] T. Amano, J. Mol. Spectrosc. 103, 436 (1984).

[23] K. P. Huber and G. Herzberg, Constants of Diatomic Molecules (Van Nostrand Reinhold, New York, 1979).
[24] N. Rosenbaum, J. Owrutsky, L. Tack, and R. Saykally, J. Chem. Phys. 84, 5308 (1986).

[25] J. Smith, J. Kim, and W. Lineberger, Phys. Rev. A 55, 2036 (1997).

[26] J. Martin, Spectrochimica Acta Part A 57, 875 (2001).

[27] C. Yamada and E. Hirota, J. Chem. Phys. 110, 2853 (1999).

[28] H. Katôx, Y. Toyasaka, and T. Suzuki, Bull. Chem. Soc. Jpn. 58, 562 (1985).

[29] E. Lee and T. Wright, J. Phys. Chem. A 107, 5233 (2003).

[30] R. Kendall, T. Dunning Jr, and R. Harrison, J. Chem. Phys. 96, 6796 (1992).

[31] J. N. Byrd, J. A. Montgomery, Jr., and R. Côté, Phys. Rev. A 82, 010502(R) (2010).

[32] J. N. Byrd, H. H. Michels, J. A. Montgomery, Jr., R. Côté, and W. C. Stwalley, J. Chem. Phys. 136, 014306 (2012).

[33] F. Weigend, F. Furche, and R. Ahlrichs, J. Chem. Phys. 119, 12753 (2003).

[34] F. Weigend and R. Ahlrichs, Phys. Chem. Chem. Phys. 7, 3297 (2005)

[35] T. Leininger, A. Nicklass, W. Küchle, H. Stoll, M. Dolg, and A. Bergner, Chem. Phys. Lett. 255, 274 (1996).

[36] D. Rappoport and F. Furche, J. Chem. Phys. 133, 134105 (2010).

[37] J. N. Byrd, R. Côté, and J. A. Montgomery, Jr., J. Chem. Phys. 135, 244307 (2011).

[38] K. Peterson and T. Dunning Jr, J. Chem. Phys. 117, 10548 (2002).

[39] It should be noted that bond lengths computed using MP2 theory differ only by a few $m \AA$ from $\operatorname{CCSD}(\mathrm{T})$ calculations using the same basis set, at a much cheaper computational cost.

[40] M. Lara, J. Bohn, D. Potter, P. Soldán, and J. Hutson, Phys. Rev. A 75, 012704 (2007).

[41] B. Roos, V. Veryazov, and P.-O. Widmark, Theor. Chem. Acc. 111, 345 (2004).

[42] J. Hill, Int. J. Quantum Chem. 113, 21 (2012).

[43] M. Douglas and N. M. Kroll, Ann. Phys. (N.Y.) 82, 89 (1974).

[44] A. Wolf, M. Reiher, and B. A. Hess, J. Chem. Phys. 117, 9215 (2002).

[45] T. Helgaker, W. Klopper, H. Koch, and J. Noga, J. Chem. Phys. 106, 9639 (1997).

[46] J. Dunham, Phys. Rev. 41, 713 (1932).

[47] A. Derevianko, W. R. Johnson, M. S. Safronova, and J. F. Babb, Phys. Rev. Lett. 82, 3589 (1999).

[48] R. D. Levine and R. B. Bernstein, Molecular Reaction Dynamics and Chemical Reactivity (Oxford University Press, New York, 1987).

[49] R. Côté and A. Dalgarno, Phys. Rev. A 62, 012709 (2000).

[50] $B_{e}=19.12087 \mathrm{~cm}^{-1}, \alpha_{e}=0.77167 \mathrm{~cm}^{-1}, \omega_{e}=3738.44$ $\mathrm{cm}^{-1}$ and $\omega_{e} x_{e}=91.42 \mathrm{~cm}^{-1}$ [24].

[51] The dipole moment function and near equilibrium potential energy curve are plotted in the supplemental material.

[52] H.-J. Werner, P. Rosmus, and E.-A. Reinsch, J. Chem. Phys. 79, 905 (1983). 
[53] The conversion from atomic units to inverse seconds used in this work is $1 /\left(2.419 \times 10^{-17} s\right)$.

[54] J. Brown and A. Carrignton, Rotational Spectroscopy of Diatomic Molecules (Cambridge, Cambridge UK, 2003). 